Check for updates

Cite this: RSC Adv., 2019, 9, 2277

Received 18th October 2018

Accepted 24th December 2018

DOI: $10.1039 / \mathrm{c} 8 \mathrm{ra0} 0652 \mathrm{~d}$

rsc.li/rsc-advances

\section{Influence of the head group on dynamics in surfactant intercalated graphite oxide $\uparrow$}

\author{
Xiaoqian Ai, ${ }^{a}$ Minmin Zhang, ${ }^{a}$ Ye Yan, ${ }^{a}$ Xiaoming Zhang, ${ }^{a}$ Xiaoxiao Cao, ${ }^{a}$ Qiong Wang ${ }^{a}$ \\ and Ligang $\mathrm{Ma} \mathbb{D}$ *b
}

The influence of the headgroup on the dynamics of three different alkylammonium cations confined in graphite oxide (GO) was studied by temperature-dependent impedance spectroscopy. X-ray diffraction, $\mathrm{X}$ ray photoelectron spectroscopy, Fourier-transform infrared spectroscopy, thermogravimetry and elemental analysis served to characterize the composites. The alkylammonium cations were connected to the $\mathrm{C}^{-} \mathrm{O}^{-}$ group of GO via ionic interactions, and the backbone of the confined molecule was distributed as a flat monolayer with the long axis parallel to the GO layer. Ngai's correlated-state model was used, with asymmetry at low temperature in the loss peaks. The calculated true activation energy of $114 \mathrm{meV} \pm 6 \%$ $\mathrm{meV}$ is almost the same as the internal rotation barrier of the alkyl macromolecule. We conclude that the relaxation process is definitely attributed to the wobbling around the long molecular axes of the confined ions, an intrinsic motion, not the reorientation of $\mathrm{C}-\mathrm{H}$ at the headgroup, and it is also due to geometric structural symmetry at the headgroup of the alkylammonium molecules with the interaction of their backbones and the skeletons of GO resulting in the difference in the apparent activation energy.

\section{Introduction}

Recently, organic-intercalated layered solids have been studied in different fields as their new physical and chemical properties are dramatically different from their bulk counterparts such as their electrical properties, ${ }^{1}$ mechanical properties, ${ }^{2}$ thermal behavior, ${ }^{3}$ surface and interfacial properties, ${ }^{4}$ and so on. Surfactants are well known for their amphipathic organic molecular structure, which can be used to modify the surface properties of hydrophilic materials, such as layered double hydroxides, ${ }^{5}$ layered metal phosphate ${ }^{6}$ and two-dimensional layered materials, ${ }^{4}$ to mix with the hydrophobic polymer chains to obtain wonderful nanocomposites. Their thermodynamics have significant practical and theoretical interest, as a result of their configurational complexity, which can be impacted by their concentration, chain length or chemical environment.

Alkylammonium halides are more common in terms of surfactants. $n$-alkylammonium halides form tetragonal crystals around room temperature belonging to space group $P 4 / \mathrm{nmm}^{-{ }^{7-9}}$ They have a two-dimensional (2D) lamellar-type structure with rod-like cations and halides anions stacked alternately along the crystallographic $\mathrm{C}_{4}$ axis. The cations are dynamically

${ }^{a}$ College of Physics and Electronic Engineering, Jiangsu Second Normal University, 210013, China

${ }^{b}$ School of Electronic Engineering, Nanjing Xiaozhuang University, 211171, Nanjing, China. E-mail: maligang186@163.com

$\uparrow$ Electronic supplementary information (ESI) available. See DOI: $10.1039 / \mathrm{c} 8 \mathrm{ra} 08652 \mathrm{~d}$ disordered and packed between the layers of anions keeping their long axis perpendicular to the layers. As the arrangement and the motion of the alkyl chains in this phase are quite similar to those in the rotator phase of the $n$-paraffins ${ }^{\mathbf{1 0}}$ below their melting point in the temperature region, this phase is called the rotator phase. Ikeda's group have proved that the rotator phase of $n$-alkylammnonium chlorides $\left(\mathrm{C}_{6}-\mathrm{C}_{10}\right)$ has uniaxial rotation of the long rod-like cations, ${ }^{7}$ and also $\mathrm{C}_{3}$ reorientation of the $\mathrm{CH}_{3}$ and $\mathrm{NH}_{3}{ }^{+}$was observed by nuclear magnetic resonance (NMR). ${ }^{89}$ The dynamical behaviors of alkylammonium cations confined in layered materials have been investigated by computational and experimental techniques, for example molecular simulation, ${ }^{\mathbf{1 1}}$ solid state NMR, ${ }^{12,13}$ Fourier-transform infrared spectroscopy (FTIR),${ }^{14}$ and so on. There have been some common conclusions that the confined alkylammonium cations assume an all-trans conformation at low temperatures and change to the gauche conformation upon heating, then leading to a phase transition at relatively elevated temperatures.

Recently, a new dynamic behavior of confined hexadecyltrimethylammonium ions in a layer was observed by Boris and coworkers ${ }^{15}$ using NMR measurements. Below room temperature, there is restricted mobility of the molecules, an essentially all-trans conformation by the chains, which has a rotator-type molecular motion around the long molecular axes of the chains. Then with increasing temperature, dynamic transformation takes place, and the confined chains undergo rotation about the molecular axis. Graphite oxide (GO), a typical pseudo-two-dimensional solid, possesses $\mathrm{C}-\mathrm{OH}$ and $-\mathrm{COOH}$ 
groups and epoxide functional groups.$^{16}$ These groups make GO easily absorb polar molecules and polar polymer by different means, and react with them to form GO intercalated nanocomposites or exfoliated nanocomposites. ${ }^{17}$ These kinds of organic-graphite oxide nano-scale hybrid materials often exhibit interesting electrochemical properties. ${ }^{18}$ What is more, Gaetano Guerra et al. ${ }^{19}$ through X-ray diffraction (XRD) and differential scanning calorimetry proved that there was a hexagonal rotator order in GO intercalation compounds at high temperatures for organic cations containing two long hydrocarbon tails. Now, we choose three different surfactants, which are hexadecyltrimethylammonium chloride $\left(\mathrm{C}_{16} \mathrm{H}_{33^{-}}\right.$ $\left.\mathrm{N}\left(\mathrm{CH}_{3}\right)_{3} \mathrm{Cl}, \mathrm{C}_{16} \mathrm{TMACl}\right)$ with three methyls on the headgroup, ethylhexadecyldimethylammonium bromide $\left(\mathrm{C}_{16} \mathrm{H}_{33} \mathrm{~N}\left(\mathrm{CH}_{3}\right)_{2}(-\right.$ $\left.\mathrm{C}_{2} \mathrm{H}_{5}\right) \mathrm{Br}, \mathrm{C}_{16} \mathrm{DMEABr}$ ) with one ethyl and two methyls on the headgroup and hexadecylpyridinium monohydrate chloride $\left(\mathrm{C}_{16} \mathrm{H}_{33} \mathrm{C}_{5} \mathrm{H}_{5} \mathrm{NCl} \cdot \mathrm{H}_{2} \mathrm{O}, \mathrm{C}_{16} \mathrm{PyCl} \cdot \mathrm{H}_{2} \mathrm{O}\right)$ with a benzene ring on its headgroup, to research whether there is $\mathrm{C}_{3}$ reorientation of the $\mathrm{CH}_{3}$ or reorientation along the molecular long axis through impedance spectroscopy. These three different surfactants have the same chain length but different headgroups, and on the basis of Ngai's correlated-state model it can be very suitable to study the nature of surfactant motion. Combining temperaturedependent dielectric relaxation spectroscopy with structural characterization, molecular level information was presented to determine the influence of headgroup on the dynamics of confined alkylammonium ions inside a GO layer.

\section{Experimental details}

\subsection{Preparation of GO}

GO was synthesized from natural graphite powder using the Hummers method ${ }^{20}$ with some modification in this paper. Graphite powder $(6 \mathrm{~g}), \mathrm{NaNO}_{3}(6 \mathrm{~g})$ and $98 \% \mathrm{H}_{2} \mathrm{SO}_{4}(132 \mathrm{~mL})$ were added into a beaker in an ice bath and stirred for $30 \mathrm{~min}$, followed by the gradual addition of $\mathrm{KMnO}_{4}$ powder $(24 \mathrm{~g})$. After another $30 \mathrm{~min}$, distilled water $(276 \mathrm{~mL})$ dropped slowly into the constantly stirred mixture of reactants, with its temperature kept below $35^{\circ} \mathrm{C}$. Then the beaker was transferred to an oil bath and stirred at $95{ }^{\circ} \mathrm{C}$ for 1 hour. Lastly, distilled water and $30 \%$ $\mathrm{H}_{2} \mathrm{O}_{2}$ were used to remove unreacted $\mathrm{KMnO}_{4}$. GO powder was obtained after the residue was washed, centrifuged, dialyzed, filtered and dried.

\subsection{Preparation of the nanocomposites}

GO powder $(100 \mathrm{mg})$ was dissolved and exfoliated for $30 \mathrm{~min}$ in $0.05 \mathrm{M} \mathrm{NaOH}$ solution $(24 \mathrm{~mL})$ inside a GA92-IID ultrasonicator (Wuxi, China), followed by the addition of $100 \mathrm{~mL}$ aqueous

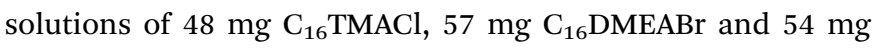
$\mathrm{C}_{16} \mathrm{PyCl} \cdot \mathrm{H}_{2} \mathrm{O}$, respectively, which is in analogy with the procedure reported by Matsuo et al. ${ }^{21}$ and Gaetano Guerra et al. ${ }^{19}$ Then the as-prepared nanocomposites were filtrated repeatedly and dried in a vacuum drying oven at $50^{\circ} \mathrm{C}$ for $12 \mathrm{~h}$, which were labelled as $\mathrm{C}_{16}$ TMA-GO, $\mathrm{C}_{16}$ DMEA-GO and $\mathrm{C}_{16} \mathrm{Py}-\mathrm{GO}$, respectively.

\subsection{Experimental techniques}

Small-angle X-ray diffraction (SAXD) patterns were performed directly on GO and nanocomposite samples using a Bruker D8 Advance diffractometer $(40 \mathrm{kV}, 40 \mathrm{~mA})$ with nickel filtered $\mathrm{Cu}-$ $K \alpha$ radiation $(\lambda=0.15406 \mathrm{~nm})$ at a scanning speed of $2^{\circ} \min ^{-1}$ in the $2 \theta$ ranges of $0.5-30^{\circ}$.

X-ray photoelectron spectroscopy (XPS) proceeded in a Thermo Scientific K-alpha that provided Al-K $\alpha$ radiation as the photon source. Survey spectra were measured with a pass energy of $200.0 \mathrm{eV}$ in $1.0 \mathrm{eV}$ steps, followed by high-resolution scans of the $\mathrm{C} 1 \mathrm{~s}$ signals in $0.10 \mathrm{eV}$ steps with a pass energy of $50.0 \mathrm{eV}$.

FTIR absorption was measured in a Nicolet Nexus 870 spectrometer on pressed $\mathrm{KBr}$ pellets under ambient conditions, over the range of 4000 to $400 \mathrm{~cm}^{-1}$. Thermogravimetric analysis (TGA) experiments proceeded under $\mathrm{N}_{2}$ atmosphere between room temperature and $1073 \mathrm{~K}$ at $20 \mathrm{~K} \mathrm{~min}^{-1}$, in a Perkin Elmer Pyris-1 calorimeter. Elemental chemical analysis was performed on a (C, H, N, S, O) Vario MICRO Elementar analyzer produced in Germany.

After these characterizations, impedance spectroscopy was conducted using an Agilent E4980A meter in a three-terminal configuration under computer control. The powders were pressed into pellets of $8 \mathrm{~mm}$ diameter and $0.4 \mathrm{~mm}$ thickness at 6.0 Mpa, and then were placed on a gold-plated copper block under vacuum inside a steel chamber, with heating at a constant rate of $1 \mathrm{~K} \mathrm{~min}^{-1}$ and measurements by a $\mathrm{Cu}-\mathrm{CuNi}$ thermocouple with an accuracy of $0.1 \mathrm{~K}$. Preliminary runs on pellets previously dried in situ under a dynamic vacuum of 30 $\mathrm{Pa}$, for $24 \mathrm{~h}$ at $50{ }^{\circ} \mathrm{C}$, prevented water effects.

\section{Results}

In order to determine the effect of surfactants on GO layer spacing, SAXD was used. Fig. 1 shows the SAXD patterns of GO and surfactant-intercalated in GO (SIGO) nanocomposites

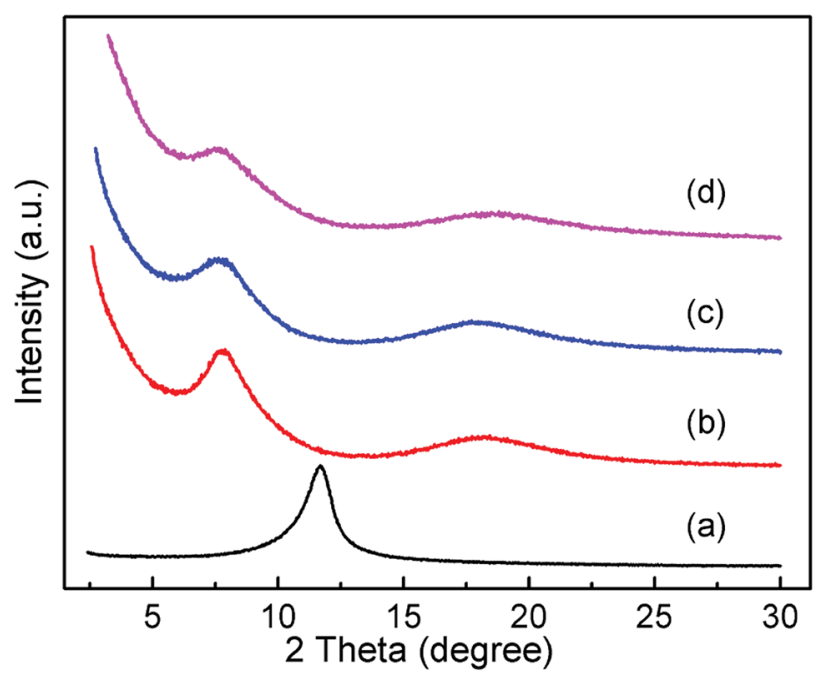

Fig. 1 SAXD patterns of (a) GO, (b) $C_{16} T M A-G O$, (c) $C_{16}$ DMEA-GO and (d) $\mathrm{C}_{16} \mathrm{Py}-\mathrm{GO}$ nanocomposites. 
starting from about $2.5^{\circ}$. The diffraction peak at $2 \theta=11.69^{\circ}$, corresponding to an interlayer spacing of $0.76 \mathrm{~nm}$, shifted to a lower angle after intercalation of surfactants with the same alkyl chain length but a different alkylammonium headgroup, which are 1.13, 1.17 and $1.16 \mathrm{~nm}$ for $\mathrm{C}_{16}$ TMA-GO, $\mathrm{C}_{16} \mathrm{Py}-\mathrm{GO}$ and $\mathrm{C}_{16}$ DMEA-GO, respectively. Their interlayer spacings are similar, and the enlarged interlayer spacing confirms that the surfactant was successfully inserted into the GO layer. From ref. 22 , the length of hexadecylamine molecules with all-trans conformations is similar to $2.2 \mathrm{~nm}$, and the interatomic distance between the farthest two hydrogen atoms of it is $0.31 \pm$ $0.12 \mathrm{~nm}$. Thus, the alkyl chains have no choice but to form a lateral monolayer with their long axis almost parallel to the GO layer. In addition, a wide diffraction peak at about $18^{\circ}$ appears compared to GO, corresponding to the characteristic peak of part reduced GO. ${ }^{23,24}$

Fig. 2 shows the XPS data of GO, $\mathrm{C}_{16}$ DMEA-GO and $\mathrm{C}_{16^{-}}$ DMEABr to determine the type of element, and others are included in the ESI. $\dagger$ We can conclude that the composite contains the elements $\mathrm{C}, \mathrm{N}$, and $\mathrm{O}$, where $\mathrm{C}$ and $\mathrm{O}$ come from $\mathrm{GO}$ and $\mathrm{N}$ comes from the surfactant. The absence of $\mathrm{Br}$ in the composite, which is at the head of the molecular chains, was attributed to the replacement of $\mathrm{Br}^{-}$by $\mathrm{C}_{-} \mathrm{O}^{-}$from the $\mathrm{GO}$ layer.

TGA was used to determine the water content in GO and its composites, as shown in Fig. 3. Combining with elemental analysis data exhibited in Table 1, we can conclude how many surfactants was intercalated into the GO layer. The TGA of GO is presented for comparison. From the derivative weight, loss of weight at each stage was obtained. GO showed one weight loss step below $160{ }^{\circ} \mathrm{C}$ associated with the desorption of adsorbed and combined water in the GO layer, and a second one associated with the decomposition of oxygen-containing functional groups. The continuous weight loss of $19 \%$ above $250{ }^{\circ} \mathrm{C}$ was attributed to the decomposition of the carbon backbone until $800{ }^{\circ} \mathrm{C} .{ }^{24}$ The composites have similar thermolysis behavior, but different from that of GO. After the surfactants were added, an additional weight loss between 200 and $480{ }^{\circ} \mathrm{C}$ was observed,

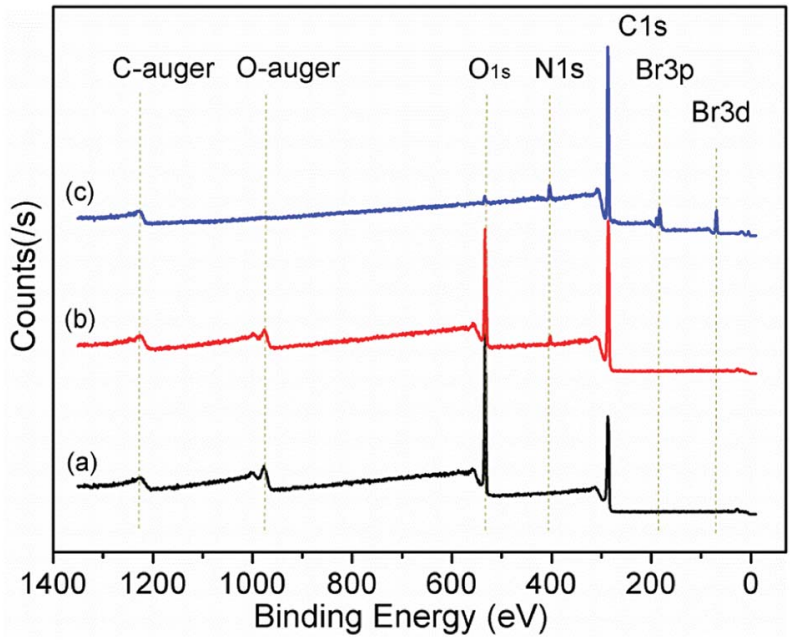

Fig. 2 Survey scans XPS spectra of (a) GO, (b) $C_{16}$ DMEA-GO and (c) $\mathrm{C}_{16} \mathrm{DMEABr}$
Table 1 The elemental analysis and water content results of $\mathrm{GO}$ and its composite materials

\begin{tabular}{lllll}
\hline Sample & $\mathrm{C}(\mathrm{wt} \%)$ & $\mathrm{H}(\mathrm{wt} \%)$ & $\mathrm{N}(\mathrm{wt} \%)$ & $\begin{array}{l}\mathrm{H}_{2} \mathrm{O} \\
\text { (weight\%) }\end{array}$ \\
\hline GO & 49.16 & 3.07 & - & 15.33 \\
$\mathrm{C}_{16}$ TMA-GO & 61.55 & 6.04 & 1.25 & 6.04 \\
$\mathrm{C}_{16}$ DMEA-GO & 62.40 & 6.32 & 1.25 & 6.87 \\
C $_{16}$ Py-GO & 63.48 & 5.57 & 1.24 & 6.21 \\
\hline
\end{tabular}

corresponding to the pyrolysis of surfactants. ${ }^{25}$ It is noteworthy that the first weight loss step in the composites suggests that only $6-7 \%$ weight loss is related to the residual water. The water content results of GO and its composite materials are listed in Table 1. Using this information and in addition to the data from the elemental analysis, nitrogen content is similar and the $\mathrm{C} / \mathrm{N}$ ratio in composites for the same added concentrations is gradually increased compared with that in the surfactant. These mean surfactant molecules exist between the GO layer. Combined with the XPS results, similar concentration values of $0.13 \mathrm{mmol}$ alkylammonium cations per $100 \mathrm{mg}$ GO were calculated in the three different nanocomposites.

In Fig. 4, one example of an FTIR spectrum of $\mathrm{C}_{16}$ DMEA-GO intercalation compounds, together with that of $\mathrm{C}_{16} \mathrm{DMEA}$ and GO are shown. The FTIR spectra of other relevant samples are included in the ESI. $\uparrow$ The wide absorption band above $3000 \mathrm{~cm}^{-1}$ is related to $\mathrm{O}-\mathrm{H}$ stretching vibrations, where the peak located at about $3600 \mathrm{~cm}^{-1}$ corresponds to the hydroxyl groups from $\mathrm{GO}^{26,27}$ Other typical absorption bands related to the vibration of $\mathrm{C}=\mathrm{O}\left(\sim 1720 \mathrm{~cm}^{-1}\right), \mathrm{C}=\mathrm{C}\left(\sim 1615 \mathrm{~cm}^{-1}\right)$, tertiary C-OH $\left(\sim 1360 \mathrm{~cm}^{-1}\right)$, phenolic groups $\left(\sim 1225 \mathrm{~cm}^{-1}\right)$ and $\mathrm{C}-\mathrm{O}-\mathrm{C}\left(\sim 1060 \mathrm{~cm}^{-1}\right)$ also appeared clearly. ${ }^{27}$ When surfactant was added into the GO layer, some new absorption bands derived from $\mathrm{C}_{16}$ DMEA related to the vibration of the $\mathrm{CH}_{2}$ asymmetric stretching vibration $\left(\sim 2920 \mathrm{~cm}^{-1}\right), v_{\text {as }}\left(\mathrm{CH}_{2}\right)$, and $\mathrm{CH}_{2}$ symmetric stretching vibration $\left(\sim 2850 \mathrm{~cm}^{-1}\right), v_{\mathrm{s}}\left(\mathrm{CH}_{2}\right)$, appeared. ${ }^{28}$ Furthermore, compared to $\mathrm{GO}$, in the nanocomposite weakening of absorption bands derived from the

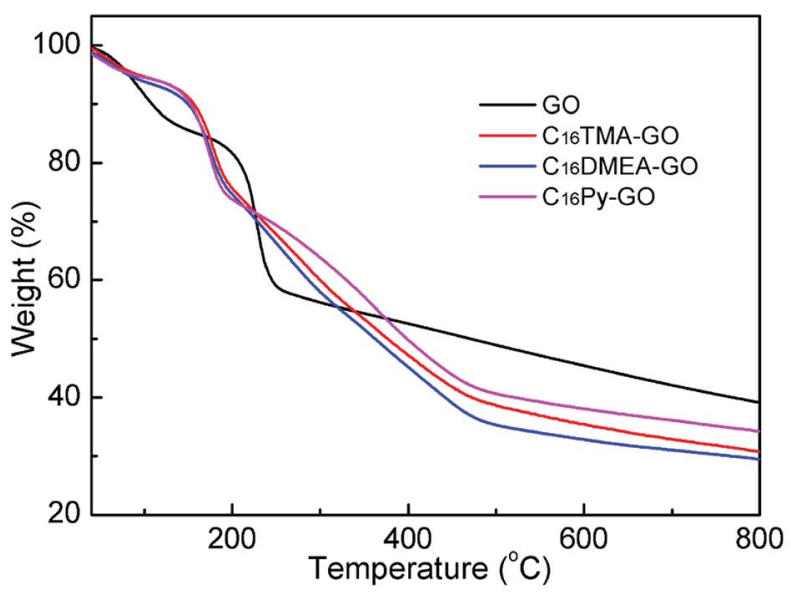

Fig. 3 TGA patterns of GO, $C_{16}$ TMA-GO, $C_{16}$ DMEA-GO and $\mathrm{C}_{16}$ PyGO nanocomposites. 


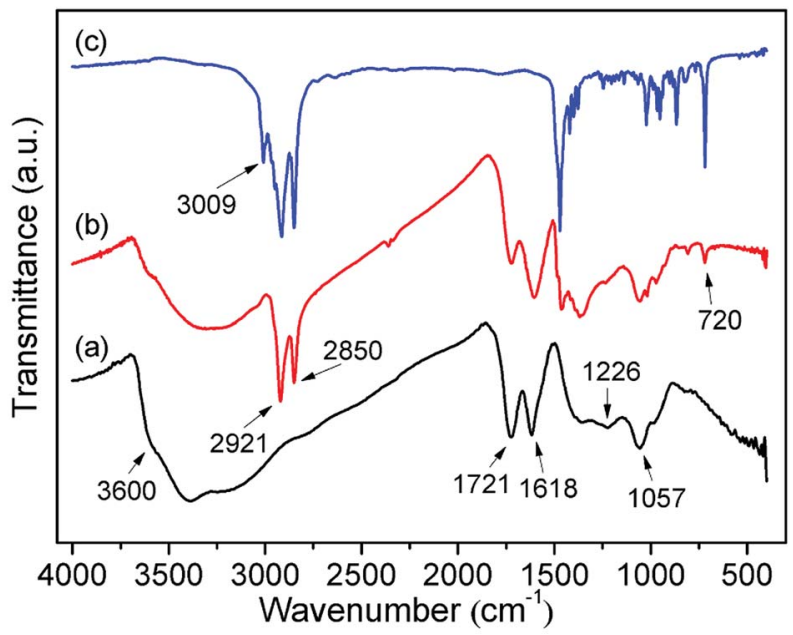

Fig. 4 FTIR spectra of (a) GO, (b) $C_{16}$ DMEA-GO and (c) $C_{16} D M E A B r$.

hydroxyl groups $\left(\sim 3600 \mathrm{~cm}^{-1}\right)$ and phenolic groups $\left(\sim 1220 \mathrm{~cm}^{-1}\right)$ indicates that the surfactant ion with a plus charge ionically bonds to the $\mathrm{C}-\mathrm{O}^{-}$groups of GO. In addition, compared to the absorption band related to the $\mathrm{C}=\mathrm{C}$ vibration $\left(\sim 1615 \mathrm{~cm}^{-1}\right)$, the intensity of the absorption band related to the $\mathrm{C}=\mathrm{O}$ vibration $\left(1720 \mathrm{~cm}^{-1}\right)$ became weak, which was attributed to the part of oxygen-containing functional groups falling off GO.

Fig. 5 shows the temperature dependence of dielectric loss at five selected frequencies for three kinds of nanocomposites. The position for the loss peak shifts toward higher temperatures with the increase of frequency, which obviously implies a relaxation process. Thermal dependence of relaxation time $\tau$ for the loss process in nanocomposites is shown in Fig. 6. The relaxation time was fitted with an Arrhenius relationship:

$$
\tau(T)=\tau_{0} \exp \left(E_{\mathrm{a}} / k T\right)
$$

where $\omega$ is the angular frequency, $\tau_{0}$ is the pre-exponential factor, $E_{\mathrm{a}}$ is the activation energy, and $k$ is the Boltzmann's constant. All the Arrhenius plots of the equilibration time show a straight line, the slope of which gives the single activation energy, as listed in Table 2 associated with other relevant parameters.

\section{Discussion}

Characterization measurements show that the alkylammonium cations were connected to the $\mathrm{C}-\mathrm{O}^{-}$group of $\mathrm{GO}$ via ionic interactions, and the backbone of the confined molecule distributes as a flat monolayer with the long axis parallel to the GO layer. Each of the nanocomposites undergoes a relaxation process, which should be attributed to the motion of the distinctive alkylammonium cation. However, from Fig. 7, it can be seen that each loss peak is obviously asymmetric with longer tails on the low temperature side, which deviate from ideal Debye characteristics. The deviation is due to Van der Waals interaction between the alkylammonium molecular backbones

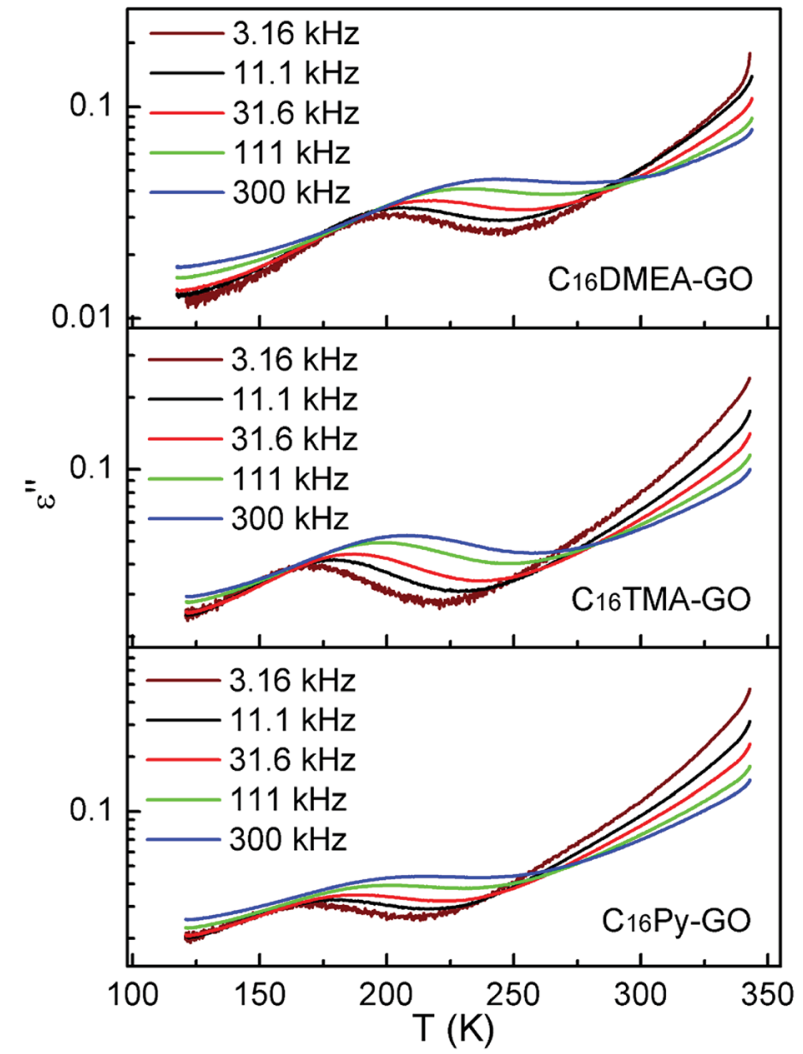

Fig. 5 Temperature dependence of dielectric loss for the nanocomposites at various frequencies.

in the alkylammonium cations, or the interaction of the alkylammonium molecular backbones with the skeletons of GO (2D polymer) in our nanocomposites. So the relaxation behavior is analyzed with the help of the Ngai's correlated-states model. ${ }^{29-31}$ A brief exposition is presented as follows.

As we know, the Debye model is used for non-interacting dipoles which follow exponential decay

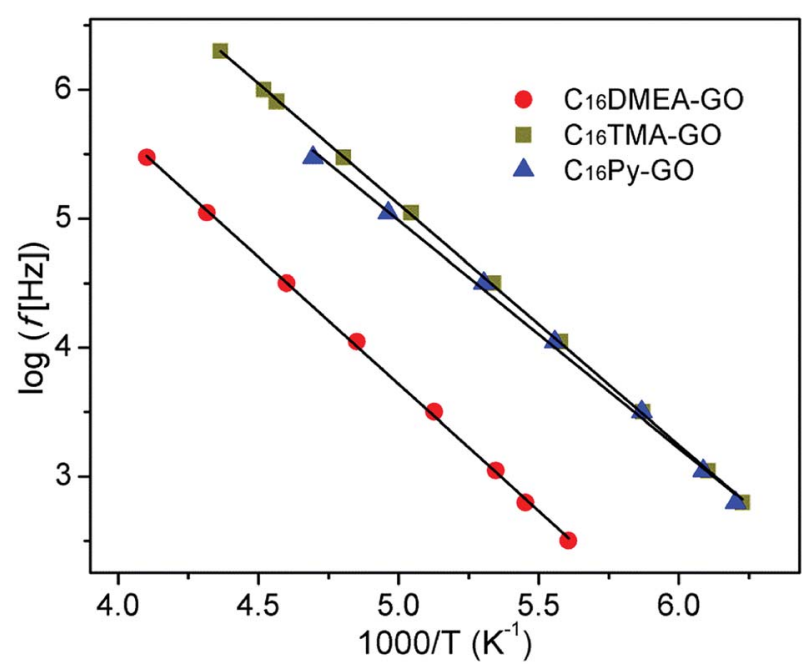

Fig. 6 Temperature dependence of relaxation time for the loss process measured at several frequencies for the nanocomposites. 
Table 2 Arrhenius parameters $E_{\mathrm{a}}^{*}$ and $\log f_{0}$ derived from relaxation processes, non-exponential factor $\beta$ and the actual activation energy $E_{a}$ calculated by eqn (4) and (5)

\begin{tabular}{lllll}
\hline Sample & $E_{\mathrm{a}}^{*}(\mathrm{meV})$ & $\log \left(f_{\mathrm{o}}[\mathrm{Hz}]\right)$ & $\beta$ & $E_{\mathrm{a}}(\mathrm{meV})$ \\
\hline $\mathrm{C}_{16}$ DMEA-GO & $391 \pm 4$ & 13.57 & 0.31 & 120 \\
$\mathrm{C}_{16}$ TMA-GO & $371 \pm 2$ & 14.45 & 0.31 & 114 \\
$\mathrm{C}_{16}$ Py-GO & $352 \pm 7$ & 13.84 & 0.32 & 107
\end{tabular}

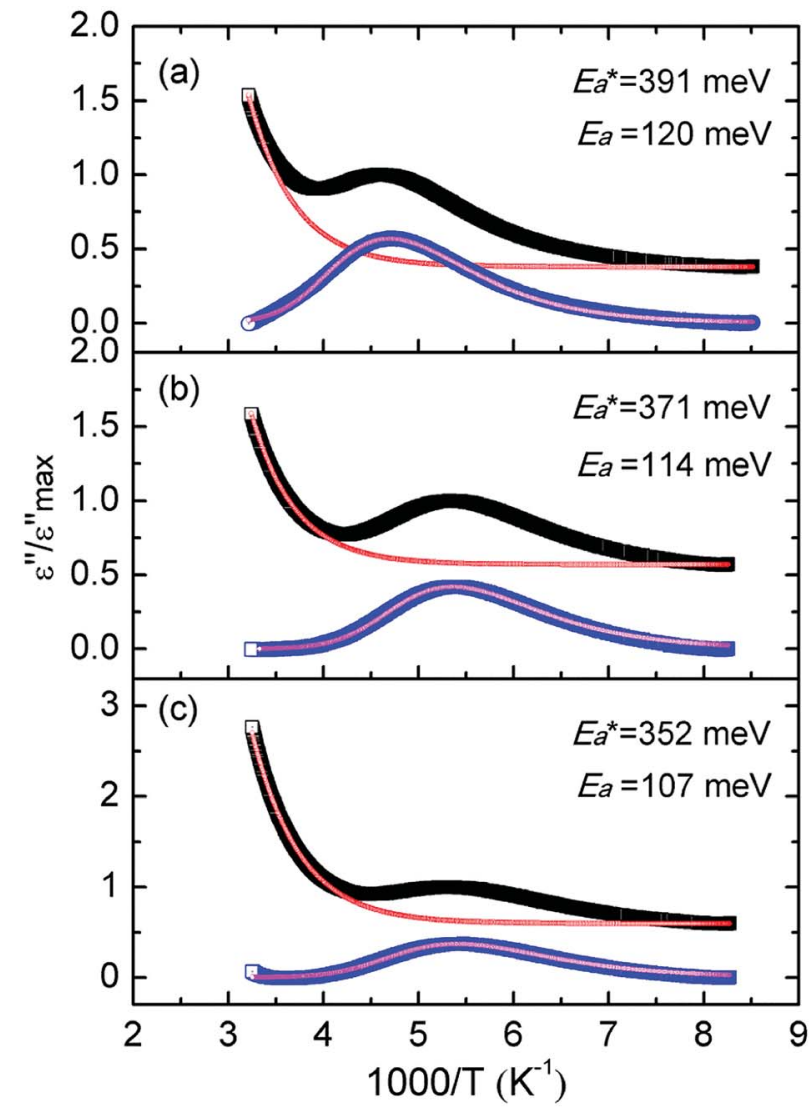

Fig. 7 Inverse temperature plots of normalized $\varepsilon^{\prime \prime}$ measured at 3.16 $\mathrm{kHz}$ before (black) and after (blue) background subtraction for (a) $\mathrm{C}_{16}$ TMA-GO, (b) $\mathrm{C}_{16}$ DMEA-GO and (c) $\mathrm{C}_{16} \mathrm{Py}-\mathrm{GO}$. Red and magenta lines represent the best fits to the backgrounds and the relaxation peaks.

$$
\psi(t)=\psi(0) \exp \left(-t / \tau_{\mathrm{D}}\right)
$$

where $\tau_{\mathrm{D}}$ is the Debye relaxation time. The model gives a symmetric peak of the energy $\operatorname{loss} \operatorname{Im}\left[\varepsilon^{*}(\omega)\right]$ in the medium. However, K. Ngai ${ }^{31}$ came to the conclusion that there exists a complicated multi-interaction for coupled dipoles, where the dipoles can no longer be treated independently, and the dielectric relaxation appears to follow the fractional exponential form

$$
\psi(t)=\mathrm{e}^{-n \gamma}\left(E_{\mathrm{c}} t\right)^{-n} \exp \left[-\left(-t / \tau_{\mathrm{p}}\right)^{1-n}\right] 0 \leq n<1
$$

where $n$ is the coupling parameter, $\gamma$ is the Euler constant, $E_{\mathrm{c}}$ is the upper cutoff of the correlated-state excitation energy, and $\tau_{\mathrm{p}}$ is a time constant, given by

$$
\tau_{\mathrm{p}}=\left[(1-n) \mathrm{e}^{n \gamma} E_{\mathrm{c}}{ }^{n} \tau_{0}\right]^{1 / 1-n}=\tau_{\infty}^{*} \exp \left(E_{\mathrm{a}}^{*} / k_{\mathrm{B}} T\right)
$$

The normalized imaginary part $\varepsilon_{n}^{\prime \prime}(\omega)$ can be expressed as

$$
\varepsilon_{n}^{\prime \prime}=\Delta z\left(\frac{1}{\pi} \int_{0}^{\infty} \mathrm{e}^{-u^{\beta}} \cos u z \mathrm{~d} u\right)
$$

Here, $\Delta$ stands for the relaxation strength; $z=\omega \tau_{\mathrm{p}}$; the integral in the bracket is the Levy function $\mathrm{Q}_{\beta}(z)$ with $u=t / \tau_{\mathrm{p}}$ and $\beta=1-n$, an increase in $\beta$ being equivalent to a decrease of the intermolecular coupling among the primitive motions. The actual activation energy $E_{\mathrm{a}}$ can be evaluated from its apparent activation energy $E_{\mathrm{a}}^{*}$ following the relationship

$$
E_{\mathrm{a}}^{*}=E_{\mathrm{a}} / \beta
$$

when $\beta<1$, i.e., $n>0$, the loss peak obtained from eqn (5) will have an asymmetric shape and its peak width increases with the decrease of $\beta . E_{\mathrm{a}}^{*}$ governs the lateral shift of the $\varepsilon^{\prime \prime}$ peaks in temperature.

Fig. 7 shows the best fitting Ngai curves to our experimental data according to the convergent expressions ${ }^{32}$ of the Levy function with various $n$. The normalized $\varepsilon^{\prime \prime}$ versus inverse temperature has been all replotted respectively. The thermal background was simulated by an exponential extrapolation and subtracted. Table 2 summarizes the nanocomposites' $E_{\mathrm{a}}^{*}, E_{\mathrm{a}}$ and $\beta$, and their true activation energy has almost similar values of $E_{\mathrm{a}}=114 \mathrm{meV} \pm$ $6 \%$, which is furthermore well consistent with the internal rotation barrier of the alkyl macromolecule. ${ }^{7,8}$ In addition, the motional correlation time $\tau$ of the restricted small-angle wobbling around the long molecular axes below room temperature is in the range of $10^{-3}$ to $10^{-4} \mathrm{~s}^{15,33}$ close to the measurement frequencies $f\left(10^{4}\right.$ to $\left.10^{6} \mathrm{~Hz}\right)$ in our dielectric experiments. So, the observed relaxation behavior is definitely attributed to the intrinsic wobbling motion of the alkylammonium cations, but not the reorientation of the $\mathrm{C}-\mathrm{H}$ at the headgroup.

The apparent activation energies of the three substances show an expected result too. From Table 1, similar concentration values of $0.13 \mathrm{mmol}$ alkylammonium cations per $100 \mathrm{mg}$ of GO were calculated in three different nanocomposites, with the chains extending in parallel to the GO layer. Thus, the interaction of the alkylammonium molecular backbones with the skeletons of GO is a major factor affecting the activation energies. $\mathrm{C}_{16} \mathrm{DMEA}^{+}$has the worst geometric structure symmetry among the three alkylammonium cations, with one ethyl and two methyls on the headgroup. $\mathrm{C}_{16} \mathrm{Py}^{+}$has the best symmetry, with a benzene ring on its headgroup. Also, the confined alkylammonium cations in layered materials assume an all-trans conformation below room temperature, ${ }^{15}$ so the difference in the size of the head groups producing different charge densities might also be a cause of the varying relaxation. Thus, the coupling was weakened, leading to a lower value of the apparent activation energy $E_{\mathrm{a}}^{*}$.

\section{Conclusions}

The loss peaks as measured using temperature-dependent impedance spectroscopy showed dielectric relaxation in the 
GO nanocomposites with the alkylammonium ions $\left(\mathrm{C}_{16} \mathrm{TMA}^{+}\right.$, $\mathrm{C}_{16} \mathrm{DMEA}^{+}, \mathrm{C}_{16} \mathrm{Py}^{+}$) confined. Asymmetry at low temperatures in the loss peaks implies a significant departure from Debye relaxation, so Ngai's correlated-state model was used, and the calculated true activation energy of $114 \mathrm{meV} \pm 6 \% \mathrm{meV}$ is almost equal to the internal rotation barrier of the alkyl macromolecule. The relaxation is definitely attributed to the wobbling around the long molecular axes of the confined ions, an intrinsic motion, not the reorientation of $\mathrm{C}-\mathrm{H}$ at the headgroup, and is also due to geometric structural symmetry at the headgroup of the alkylammonium molecules resulting in the difference in the apparent activation energy.

\section{Conflicts of interest}

There are no conflicts to declare.

\section{Acknowledgements}

This work was supported by the Natural Science Foundation of the Jiangsu Higher Education Institutions of China (17KJB140005, 17KJB140012), the Natural Science Foundation of China (11804125), the College Students' Innovative Training Program of Jiangsu Province (201814436003Z), and by the Natural Science Foundation of Nanjing Xiaozhuang University (2016NXY10).

\section{References}

1 S. Konwer, A. Begum, S. Bordoloi and R. Boruah, Expanded graphene-oxide encapsulated polyaniline composites as sensing material for volatile organic compounds, J. Polym. Res., 2017, 24, 37.

2 Q. Tu, I. Spanopoulos, S. Hao, C. Wolverton, M. G. Kanatzidis, G. S. Shekhawat and V. P. Dravid, Out-ofPlane Mechanical Properties of 2D Hybrid OrganicInorganic Perovskites by Nanoindentation, ACS Appl. Mater. Interfaces, 2018, 10, 22167-22173.

3 Q. Kong, H. Zhang, L. Zheng, D. Y. Wang and J. Zhang, Effect on thermal and combustion behaviors of montmorillonite intercalation nickel compounds in polypropylene/IFR system, Polym. Adv. Technol., 2017, 28, 965-970.

4 S. Zhang, Q. Liu, H. Cheng, F. Gao, C. Liu and B. J. Teppen, Thermodynamic Mechanism and Interfacial Structure of Kaolinite Intercalation and Surface Modification by Alkane Surfactants with Neutral and Ionic Head Groups, J. Phys. Chem. C, 2017, 121, 8824-8831.

5 B. Zhang, Z. Dong, D. Sun, T. Wu and Y. Li, Enhanced adsorption capacity of dyes by surfactant-modified layered double hydroxides from aqueous solution, J. Ind. Eng. Chem., 2017, 49, 208-218.

$6 \mathrm{X}$. Han, H. Yong and D. Sun, Tuning Tribological Performance of Layered Zirconium Phosphate Nanoplatelets in Oil by Surface and Interlayer Modifications, Nanoscale Res. Lett., 2017, 12, 542.

7 S. Iwai, M. Hattori, D. Nakamura and R. Ikeda, Ionic dynamics in the rotator phase of n-alkylammonium chlorides (C6-C10), studied by $1 \mathrm{H}$ nuclear magnetic resonance, electrical conductivity and thermal measurements, J. Chem. Soc., Faraday Trans., 1993, 89, $827-831$.

8 S. Iwai, R. Ikeda and D. Nakamura, $1 \mathrm{H}$ nuclear magnetic resonance, differential scanning calorimetry, and electrical conductivity studies on the phase transitions of pentylammonium chloride and the cationic self-diffusion in its rotator phase, Can. J. Chem., 1988, 66, 1961-1969.

9 S.-i. Fukada, H. Yamamoto, R. Ikeda and D. Nakamura, Hydrogen-1 nuclear magnetic resonance, differential thermal analysis, X-ray powder diffraction and electrical conductivity studies on the motion of cations, including self-diffusion in crystals of propylammonium chloride and bromide as well as their n-deuterated analogues, J. Chem. Soc., Faraday Trans. 1, 1987, 83, 3207-3222.

10 A. Müller, An X-ray investigation of normal paraffins near their melting points, Proc. R. Soc. London, Ser. A, 1932, 514-530.

11 V. V. Naik and S. Vasudevan, Effect of Alkyl Chain Arrangement on Conformation and Dynamics in a Surfactant Intercalated Layered Double Hydroxide: Spectroscopic Measurements and MD Simulations, J. Phys. Chem. C, 2011, 115, 8221-8232.

12 R. Guegan, E. Véron, L. F. Le, M. Ogawa and S. Cadars, Structure and Dynamics of Nonionic Surfactant Aggregates in Layered Materials, Langmuir, 2017, 33, 9759.

13 X. Q. Ai and L. G. Ma, Study of conformation and dynamic of surfactant molecules in graphite oxide via NMR, Appl. Phys. A: Mater. Sci. Process., 2016, 122, 1-7.

$14 \mathrm{Y}$. L. And and $\mathrm{H}$. Ishida, Concentration-Dependent Conformation of Alkyl Tail in the Nanoconfined Space: Hexadecylamine in the Silicate Galleries, Langmuir, 2003, 19, 2479-2484.

15 B. B. Kharkov, R. W. Corkery and S. V. Dvinskikh, Phase Transitions and Chain Dynamics of Surfactants Intercalated into the Galleries of Naturally Occurring Clay Mineral Magadiite, Langmuir, 2014, 30, 7859-7866.

16 W. Gao, L. B. Alemany, L. Ci and P. M. Ajayan, New insights into the structure and reduction of graphite oxide, Nat. Chem., 2009, 1, 403-408.

17 G. Sahoo, N. Sarkar and S. K. Swain, The effect of reduced graphene oxide intercalated hybrid organoclay on the dielectric properties of polyvinylidene fluoride nanocomposite films, Appl. Clay Sci., 2018, 162, 69-82.

18 A. Ambrosi and M. Pumera, Electrochemically Exfoliated Graphene and Graphene Oxide for Energy Storage and Electrochemistry Applications, Chemistry, 2016, 22, 153-159.

19 M. Mauro, M. Maggio, V. Cipolletti, M. Galimberti, P. Longo and G. Guerra, Graphite oxide intercalation compounds with rotator hexagonal order in the intercalated layers, Carbon, 2013, 61, 395-403.

$20 \mathrm{~W}$. S. Hummers Jr and R. E. Offeman, Preparation of graphitic oxide, J. Am. Chem. Soc., 1958, 80, 1339.

21 Y. Matsuo, T. Niwa and Y. Sugie, Preparation and characterization of cationic surfactant-intercalated graphite oxide, Carbon, 1999, 37, 897-901. 
22 X. Fan and K. Zhao, Aggregation behavior and electrical properties of amphiphilic pyrrole-tailed ionic liquids in water, from the viewpoint of dielectric relaxation spectroscopy, Soft Matter, 2014, 10, 3259-3270.

23 H.-K. Jeong, H.-J. Noh, J.-Y. Kim, M. Jin, C. Park and Y. Lee, X-ray absorption spectroscopy of graphite oxide, Europhys. Lett., 2008, 82, 67004.

24 W. Li, X.-Z. Tang, H.-B. Zhang, Z.-G. Jiang, Z.-Z. Yu, X.-S. Du and Y.-W. Mai, Simultaneous surface functionalization and reduction of graphene oxide with octadecylamine for electrically conductive polystyrene composites, Carbon, 2011, 49, 4724-4730.

25 F. Kooli, L. I. Mianhui, S. F. Alshahateet, F. Chen and Y. Zhu, Characterization and thermal stability properties of intercalated Na-magadiite with cetyltrimethylammonium (C16TMA) surfactants, J. Phys. Chem. Solids, 2006, 67, 926931.

26 T. Szabó, O. Berkesi, P. Forgó, K. Josepovits, Y. Sanakis, D. Petridis and I. Dékány, Evolution of surface functional groups in a series of progressively oxidized graphite oxides, Chem. Mater., 2006, 18, 2740-2749.
27 A. B. Bourlinos, D. Gournis, D. Petridis, T. Szabó, A. Szeri and I. Dékány, Graphite oxide: chemical reduction to graphite and surface modification with primary aliphatic amines and amino acids, Langmuir, 2003, 19, 6050-6055.

28 E. Fuente, J. Menendez, M. Diez, D. Suarez and M. MontesMoran, Infrared spectroscopy of carbon materials: a quantum chemical study of model compounds, J. Phys. Chem. B, 2003, 107, 6350-6359.

29 K. Ngai, C. White and A. Jonscher, On the origin of the universal dielectric response in condensed matter, Nature, 1979, 277, 185-189.

30 K. Ngai and C. White, Frequency Dependence of Dielectric Loss in Condensed Matter, Phys. Rev. B, 1979, 20, 2475.

$31 \mathrm{~K}$. Ngai, Unified theory of 1f noise and dielectric response in condensed matter, Phys. Rev. B, 1980, 22, 2066.

32 E. W. Montroll and J. T. Bendler, On Lévy (or stable) distributions and the Williams-Watts model of dielectric relaxation, J. Stat. Phys., 1984, 34, 129-162.

33 B. B. Kharkov and S. V. Dvinskikh, Conformational Dynamics of Surfactant in a Mesolamellar Composite Studied by Local Field NMR Spectroscopy, J. Phys. Chem. C, 2013, 117, 24511-24517. 